Suizidprävention ist Alltagsarbeit für Psychiaterinnen und Psychiater; auch Hausärztinnen und Hausärzte sind hier immer wieder gefordert. Suizidprävention ist aber auch eine Aufgabe der ganzen Gesellschaft. In der Schweiz ist die Suizidrate nach wie vor hoch: Es braucht politische Entscheide auf vielen Ebenen, damit gefährdete Menschen im entscheidenden Moment ihrer Krise leichter Unterstützung finden als Zugang zu Suizidmitteln. Ein Blick auf Europa im Zeichen der Eurokrise bringt diesbezüglich Schreckliches - und Erstaunliches zutage.

\title{
Wirtschaftskrise und psychische Gesundheit
}

In der Diskussion um Suizidprävention begegnet man immer wieder der fatalistischen Meinung, wer sich umbringen will, der findet ein Mittel. Die Wissenschaft spricht hier eine andere Sprache: Die internationale Evidenz zeigt klar auf, dass beispielsweise die Beschränkung des Zugangs zu Suizidmitteln die Suizidrate signifikant senken kann. Möglichkeiten der Suizidprävention sind nicht erst seit der Einführung von flächendeckenden Fortbildungen für Grundversorger [1] (zur Früherkennung von Krisen und Suizidalität oder der über ganz Europa verbreiteten Präventionskampagne «Bündnis gegen Depression» oder etwa der verbesserten Medienberichterstattung bekannt. Die Stadt Bern hat eines der besten Beispiele zur Beschränkung der Mittel durchgeführt: die Sicherung der Münsterplattform durch Netze. Negativen Unkenrufen zum Trotz stiegen die Suizidraten an den nahegelegenen Brücken nicht an - im Gegenteil, sie sanken ebenfalls. Ein treffendes Beispiel dafür, dass Menschen, die an Suizid denken, eigentlich gar nicht sterben wollen, jedoch «sO» nicht mehr weiterleben können.

\section{Wirtschaftskrisen verschlechtern die psychische Gesundheit der Bevölkerung.}

In einem ganz anderen Ausmass zeigt sich zurzeit die Suizidproblematik in Zusammenhang mit der Eurokrise, die ganz Europa durchschüttelt [2, 3]. Die Anzahl Suizide von Menschen, die jünger sind als 65 Jahre, sind seit 2007 in der gesamten EU wieder im Steigen begriffen. Griechenland verzeichnete zwischen Januar und Mai 2011 einen Anstieg der Suizidraten von 40\%. Die Auswirkungen politischer Sparmassnahmen auf den dortigen Gesundheitssektor sind gewaltig: Seit 2011 gingen 150000 Arbeitsplätze verloren; 15\% der Spitalkosten wurden eingespart, Ärztetarife um 25\% gekürzt und allgemeine Minimallöhne um 20\%.

Dass Arbeitslosigkeit auf die psychische Gesundheit einen Einfluss hat, ist allseits bekannt. 34\% der Arbeitslosen haben psychische Probleme, bei Personen mit einer gesicherten Arbeitsstelle sind es 16\%. In Spanien stieg zwischen 2006 und 2010 die Prävalenz psychischer Störungen signifikant an; den höchsten Anstieg verzeichnete die Major Depression. Jüngste Studien bringen dies in Zusammenhang mit latenter Arbeitslosigkeit oder dem Verlust des Arbeitsplatzes.
Finnland und Schweden sind vorbildlich in der Krisenbewältigung: Trotz steigenden Arbeitslosenzahlen sanken dort die Suizidraten weiterhin kontinuierlich. Beide Länder führten und führen während der Wirtschaftskrise intensive soziale Unterstützungsprogramme für Arbeitslose durch. Auch hier konnten diejenigen Länder mit guten Unterstützungsprogrammen ein Ansteigen verhindern oder auffangen.

\section{Länder, die trotz Krise ihre soziale}

Verantwortung wahrnehmen, verzeichnen eine deutlich niedrigere Suizidrate.

Island - eines der Länder, welches die Krise auf sehr heftige Art und Weise zu spüren bekam, weigerte sich standhaft, im Bereich Gesundheits- und Sozialwesen übermässige Einsparungen vorzunehmen. Arbeitslose erhielten aktive Unterstützung bei der Wiedereingliederung.

Der Einfluss politischer Entscheidungen auf die Gesundheit der Bevölkerung ist nicht neu. Es ist an uns, der Ärzteschaft und den Gesundheitsorganisationen, hellhörig zu sein für diese Zusammenhänge und Tatsachen und - als ein ceterum censeo - die Politik auf die Folgen für die Bevölkerung - für unsere Patientinnen und Patienten - hinzuweisen. Sieht man die aktuellen Schweizer Suizidstatistiken, haben wir hier noch Arbeit vor uns - vor allem in der Prävention von Alterssuizid.

Barbara Weil, Leiterin Ressort Gesundheitsförderung und Prävention

\section{Literatur}

1 Rutz W, Wålinder J, Eberhard G, Holmberg G, von Knorring $\mathrm{AL}$, von Knorring $\mathrm{L}$, et al. An educational program on depressive disorders for general practitioners on Gotland: background and evaluation. Acta psychiatrica Scandinavica. 1989;79(1):19-26.

2 Karanikolos M, Mladovsky P, Cylus J, Thomson S, Basu S, Stuckler D, Mackenbach JP9. Financial crisis, austerity, and health in Europe: Lancet (Published Online) March 27;2013.

3 Mackenbach JP, Karanikolos M, McKee M. The unequal health of Europeans: successes and failures of policies. Lancet. 2013;381:1125-34. Published Online, March 27:2013. 